\title{
Die Angleichung der Verkehrsinfrastruktur im vereinigten Deutschland zwischen 1990 und 1999
}

\section{Overcoming Disparities in Transport Infrastructure in the Reunited Germany between 1990 and 1999}

\begin{abstract}
Kurzfassung
Die Güte der Verkehrsinfrastruktur hat erheblichen Einfluss auf die wirtschaftliche Entwicklung von Regionen. Ist sie unzureichend - wie in der ehemaligen DDR zum Zeitpunkt der Wiedervereinigung -, so kann sie zum wachstumslimitierenden Faktor werden. Deshalb war und ist es das Ziel der Politik, für eine Verbesserung zu sorgen. Aufgabe dieses Beitrags ist es, zu überprüfen, inwieweit dies in der Zwischenzeit gelungen ist. Dabei lässt sich für die Schiene die Aussage machen, dass die Anbindungsqualität der Kreise in den neuen Bundesländern im Durchschnitt bereits besser als in den alten Bundesländern ist, wobei allerdings die Unterschiede zwischen Stadt und Land erheblich zugenommen haben. Dagegen liegt der Aus- und Neubau von Straßen noch deutlich zurück.
\end{abstract}

\begin{abstract}
The quality of transport infrastructure has a major impact on the economic development of regions. Where it is inadequate - as it was in the former GDR at the time of reunification - it can act as a factor stifling growth. For politics, the goal therefore was at that time - and still remains - to improve on the situation found. This article attempts to provide an appraisal of the success to date of attempts to bring about this improvement. As far as rail transport is concerned, counties in the "new Länder" (of the former East Germany) now on average have better connections to the network than counties in western Germany. However, disparities between urban and rural areas have increased considerably. By contrast, relatively little progress has been made so far in up-grading and adding to the road network.
\end{abstract}

\section{Einleitung}

Aufgabenstellung der Untersuchung

Die Öffnung der Grenzen nach Osten und die deutsche Wiedervereinigung haben die Verkehrspolitik vor erhebliche Aufgaben gestellt, die vor allem auf einer Veränderung der Verkehrsströme, der Unterbrechung von Verkehrsverbindungen zwischen Ost und West nach dem Zweiten Weltkrieg und einer vollkommen veralteten Verkehrsinfrastruktur in der ehemaligen DDR basieren. Diese Defizite behindern das Zusammenwachsen Deutschlands und Europas und erschweren den wirtschaftlichen Aufschwung im Osten erheblich, da die Verkehrsinfrastruktur einen der wichtigsten Standortfaktoren im interregionalen Wettbewerb um
Investitionen darstellt. Vor diesem Hintergrund war es verständlich und vernünftig, dass die Bundesregierung der Verbesserung der Verkehrsinfrastruktur innerhalb der neuen Bundesländer, aber auch zwischen Ost und West eine erhebliche Aufmerksamkeit gewidmet hat und immer noch widmet. Dieser hohe Stellenwert äußert sich u.a. in Plänen (Bundesverkehrswegeplan) und Programmen (z.B. Investitionsprogramm für den Ausbau der Bundesschienenwege, Bundesfernstraßen und Bundeswasserstraßen in den Jahren 1990 bis 2002), hat aber auch bereits zu erheblichen Investitionen ins Verkehrswegenetz in den 90er Jahren geführt. 
Aufgabe der folgenden Untersuchung ist es, zu untersuchen, inwieweit die schon durchgeführten Investitionen zu einer Angleichung der Leistungsfähigkeit der Verkehrsinfrastruktur in Ost und West geführt haben und wo noch Defizite bestehen; dabei erfolgt eine Konzentration auf den Personen- im Rahmen des Straßenund Schienenverkehrs.

\section{Verwendete Untersuchungsmethoden}

Will man die von verkehrlichen Maßnahmen ausgehenden Wirkungen beurteilen, so bedarf es hierzu eines geeigneten Programmsystems; ein solches ist am Fachgebiet „Empirische Wirtschaftsforschung“ der Universität Gh Kassel $^{1}$ entwickelt worden und trägt den Namen VERENA.

VERENA ist ein komplexes Simulationsmodell, mit dessen Hilfe der gesamte Güter- und Personenverkehr (auf Straße, Schiene, in der Luft und auf dem Wasser) analysiert und prognostiziert werden kann. Seine Bestandteile sind digitalisierte Verkehrswegenetze, in diese Netze eingepasste Verkehrsregionen und Algorithmen, die es erlauben, eine Vielzahl von raumordnungspolitisch, verkehrspolitisch und umweltpolitisch relevanten Indikatoren $\mathrm{zu}$ berechnen.

Die mit Hilfe von VERENA berechneten Ergebnisse bauen auf dem der Untersuchung zu Grunde gelegten Verkehrswegenetz, den Standorten in diesem Netz sowie den verwendeten Lösungsalgorithmen auf. $\mathrm{Zu}$ diesen drei Elementen sind folgende Anmerkungen zu machen:

Für die Bundesrepublik Deutschland wurden alle überörtlich wichtigen Straßen aufgenommen, so alle Bundesautobahnen, Bundesstraßen und darüber hinaus einige Landstraßen. Für die Kanten sind eine Reihe von Merkmalen entwickelt worden, so etwa ihre Länge und Leistungsfähigkeit (in Abhängigkeit von der Anzahl der Spuren, Steigung und Gefälle, Kurven, Ortsdurchfahrten usw.). Für jede Kante existiert entsprechend eine Widerstandsfunktion, die angibt, welche Geschwindigkeit auf Grund der Art der Straße und ihrer Auslastung gefahren werden kann. Das Eisenbahnnetz in Deutschland wurde komplett erfasst, sofern es zz. für Personen- und (oder) Güterverkehr genutzt wird. Die Fahrzeit zwischen den Ursprungs- und Zielorten muss in diesem Netz nicht, wie auf der Straße, über Widerstandsfunktionen geschätzt werden, sondern kann den Fahrplänen der Eisenbahngesellschaften entnommen werden.

In die digitalisierten Verkehrswegenetze sind Standorte eingepasst, die die sie umgebenden Regionen repräsentieren. In Deutschland sind dies die 440 Stadt- und
Landkreise. Die digitalisierten Verkehrswegenetze lassen die Bestimmung von Fahrzeiten und -geschwindigkeiten in Abhängigkeit von der Belegung der Verkehrswege zwischen allen im Modell berücksichtigten Standorten zu. Mit ihrer Hilfe können Indikatoren berechnet werden, die sich auf den heutigen Zustand des Verkehrswegenetzes beziehen können, aber auch auf einen fiktiven Zustand, der sich dann einstellen wird, wenn sich verkehrsrelevante Rahmenbedingungen ändern. In den folgenden Ausführungen stellt das Straßen- und Eisenbahnnetz zum Zeitpunkt der Grenzöffnung den Bezugsfall dar; der Analysefall wird durch den Zustand des Jahres 1999 gebildet. Berechnet man die Ausprägung der Indikatoren im Bezugs- und im Analysefall, so lässt sich durch eine Differenzenbildung ermitteln, welche Effekte von den einzelnen und allen durchgeführten Vorhaben ausgehen. Aus der Menge der mit Hilfe von VERENA berechenbaren Indikatoren sind zwei ausgewählt worden, nämlich die Güte der Verkehrsinfrastruktur und die Verbindungsqualität zwischen Oberzentren.

Die Güte der Verkehrsinfrastruktur lässt sich sinnvoll über die Luftliniengeschwindigkeit zu allen übrigen Regionen ,des Gesamtraums berechnen ${ }^{2}$, die im Algorithmus mit ihrer Bedeutung (positiv) und ihrer Entfernung (negativ) gewichtet werden (gravitationstheoretischer Ansatz). Bei den Verkehrsinfrastrukturindikatoren macht es Sinn, nicht nur summarische Werte darzustellen, die durchschnittliche Verbindungsqualitäten $\mathrm{zu}$ allen Zielorten im Untersuchungsraum beinhalten, sondern auch auf einzelne ausgewählte Quell-Ziel-Beziehungen einzugehen. Von besonderem Interesse sind dabei die schlechten Verbindungen, da sie Hinweise darauf geben, welche Quell-Ziel-Beziehungen bevorzugt verbesserungsbedürftig sind. Damit eine Vergleichbarkeit gewährleistet ist, erfolgt dabei eine Konzentration auf die Verbindungen zwischen Oberzentren.

Zur leichteren Interpretation der Ergebnisse und aus Gründen der politischen Relevanz werden die auf der Ebene von Stadt- und Landkreisen errechneten Ergebnisse zu Landesergebnissen zusammengefasst. Die Länderergebnisse sind dabei das gewichtete arithmetische Mittel jener Stadt- und Landkreise, die dem entsprechenden Bundesland angehören, wobei die Gewichte durch die Einwohnerzahl der Kreise gebildet werden. Da die Bundesländer ganz unterschiedliche Größe und Verstädterung besitzen, macht es Sinn, Hamburg und Schleswig-Holstein, Bremen und Niedersachsen, Berlin und Brandenburg sowie das Saarland und Rheinland-Pfalz zu jeweils größeren regionalen Untersuchungseinheiten zusammenzufassen, um eine vernünftige interregionale Vergleichbarkeit zu gewährleisten. 


\section{Verkehrsinfrastruktur und Regionalentwicklung}

\subsection{Ein Überblick}

Eine gute Verkehrsinfrastruktur erhöht die Effizienz der im Produktionsprozess eingesetzten Faktoren Arbeit und Kapital und wirkt damit für die privaten Unternehmen kostensenkend; sie ist deshalb ein entscheidender Standortfaktor beim Wettbewerb zwischen Regionen um Investitionen und damit um Arbeitsplätze. Will man herausfinden, welchen Einfluss die Güte der Verkehrsinfrastruktur auf den Entwicklungsstand und seine Veränderung in einer Region nimmt, so stehen unterschiedliche methodische Ansätze zur Ermittlung dieses funktionalen Zusammenhanges zur Verfügung; sie bestehen in Unternehmensbefragungen (Ermittlung, was Unternehmen meinen und sagen) und/oder ökonometrischen Modellen (Ermittlung, was Unternehmen tun). Obwohl empirische Untersuchungen nicht $\mathrm{zu}$ gleichen Ergebnissen führen ${ }^{3}$, lassen sie jedoch in ihrer überwiegenden Anzahl folgende Schlussfolgerungen zu:

- Die internationale Standortkonkurrenz um Investitionen und Arbeitsplätze wird härter. Standortdefizite werden heftiger und schneller bestraft als in der Vergangenheit.

- Die Güte der Verkehrsinfrastruktur spielt bei der internationalen Standortwahl eine wichtige Rolle. Wegen der Globalisierung der Märkte, verbunden mit einer abnehmenden Fertigungstiefe und dem Wunsch nach just-in-time-Lieferung ist sogar mit einer weiteren Aufwertung der Verkehrswege als bestimmende Standortfaktoren zu rechnen.

- Westdeutschland besitzt bei der Verkehrsinfrastruktur einen komparativen Wettbewerbsvorteil gegenüber der internationalen Konkurrenz, den es zu erhalten gilt (der allerdings Nachteile bei anderen Standortfaktoren immer weniger ausgleichen kann).

- Die entgegengesetzte Aussage gilt für die neuen Bundesländer. Eine unzureichende Verkehrsinfrastruktur stellt für sie - wie im Folgenden zu belegen sein wird - ein erhebliches Investitionshindernis dar.

\subsection{Zur Bedeutung einer unzureichenden Verkehrsinfrastruktur als Investitionshemmnis in den neuen Bundesländern}

Das Deutsche Institut für Wirtschaftsforschung 4 hat für das Jahr 1990 Angaben zum Anlagevermögen (Ausrüstungen und Bauten) im Bereich der Verkehrsinfrastruktur für West- und Ostdeutschland vorgelegt. Schätzungen für Ostdeutschland mögen eine gewisse Ungenauigkeit dieser Zahlen beinhalten, ermöglichen aber einen prinzipiellen Vergleich. Tabelle 1 bringt den Modernitätsgrad der Verkehrsinfrastruktur 1990 zum Ausdruck, der als Nettoanlagevermögen (Bruttoanlagevermögen - Abschreibungen), dividiert durch das Bruttoanlagevermögen, definiert ist. Je höher die entsprechende Ausprägung, umso moderner die vorhandene Verkehrsinfrastruktur, da mit steigendem Alter ein immer größerer Teil der getätigten Investitionen abgeschrieben ist.

Bei der Verkehrsinfrastruktur insgesamt lag der Modernitätsgrad 1990 in Westdeutschland bei $70,7 \%$, jener in Ostdeutschland bei $50,9 \%$; dies ist ein deutlicher Hinweis auf den stark überalteten Kapitalstock im Verkehr in der ehemaligen DDR. Die Verkehrsinfrastruktur insgesamt lässt sich in Verkehrswege und Umschlagplätze aufteilen, wobei beide Komponenten sich nach Verkehrsträgern unterscheiden lassen. Das Defizit Ostdeutschlands zeigt sich bei jeder dieser betrachteten Teilelemente der Verkehrsinfrastruktur.

Tabelle 1

Modernitätsgrad der Verkehrsinfrastruktur 1990

\begin{tabular}{|l|c|c|c|}
\hline & West & Ost & Insgesamt \\
\hline Verkehrsinfrastruktur insgesamt & 70,66 & 50,90 & 68,54 \\
Verkehrswege & 71,87 & 51,31 & 69,61 \\
Eisenbahn, S-Bahn & 61,81 & 49,44 & 59,74 \\
Stadtschnellbahn, Straßenbahn & 87,16 & 52,21 & 85,83 \\
Straßen und Brücken & 73,70 & 52,50 & 71,73 \\
darunter Bundesfernstraßen & 77,05 & 55,33 & 74,66 \\
Wasserstraßen & 66,42 & 51,12 & 63,71 \\
Rohrfernleitungen & 46,80 & 41,43 & 45,27 \\
Umschlagplätze & 59,50 & 45,73 & 58,36 \\
Eisenbahn, S-Bahn & 52,10 & 42,45 & 50,88 \\
Binnenhäfen & 57,59 & 47,13 & 57,02 \\
Seehäfen & 65,76 & 54,47 & 65,07 \\
Flughäfen & 68,27 & 54,79 & 67,94 \\
\hline
\end{tabular}

Quelle: Eigene Berechnungen nach DIW, a.a.O.

Die hohe Bedeutung der Verkehrsinfrastruktur als Standortmotiv und ihre vollkommen unzureichende Quantität und Qualität im Osten ließ und lässt erwarten, dass Infrastrukturmängel ein erhebliches Hemmnis bei der wirtschaftlichen Entwicklung der neuen Bundesländer darstellten und immer noch darstellen. Diese Hypothese bestätigt sich bei einer Auswertung von Unternehmensbefragungen, die vor allem vom ifoInstitut durchgeführt worden sind. So stellten Infrastrukturmängel zusammen mit Bewertungsfragen den Hauptgrund dafür dar, dass westdeutsche Unternehmen gar nicht oder nicht im ursprünglich geplanten Umfang in den neuen Bundesländern investiert haben. Dieses Defizit wurde vor allem von großen Industrieunternehmen als sehr störend empfunden. ${ }^{5}$ Fragt man nach, welche Infrastrukturengpässe vor allem für die Investitionszurückhaltung verantwortlich sind, so la- 
gen 1990 die Infrastrukturbereiche Telekommunikation und Straßenverkehr deutlich an der Spitze. Mehr als $50 \%$ der befragten Unternehmen sahen für sie eine höchste Dringlichkeit für eine Verbesserung an; eine gleiche Einschätzung nahmen $19 \%$ der Unternehmen für den Schienenverkehr und $7 \%$ für den Luftverkehr vor, die damit eine deutlich geringere Dringlichkeit als der Straßenverkehr aufwiesen. ${ }^{6}$

\subsection{Politische Maßnahmen zur Verbesserung der unzureichenden Verkehrsinfrastruktur}

Gemäß der hohen Wichtigkeit einer akzeptablen Verkehrsinfrastruktur für die wirtschaftliche Entwicklung im Osten sind erhebliche Investitionen in diesem Bereich vorgenommen worden und weiterhin vorgesehen. So lagen die Bruttoanlageinvestitionen in die Verkehrsinfrastruktur je Einwohner in den ersten fünf Jahren des letzten Jahrzehnts in Ostdeutschland mehr als doppelt so hoch wie in Westdeutschland. ${ }^{7}$ Während die Verkehrsinfrastruktur in Westdeutschland in den 90er Jahren im Durchschnitt älter geworden ist, hat sich der Modernisierungsgrad im Osten deutlich verbessert.

Die Politik ist gewillt, diese überproportionalen Anstrengungen für den Osten auch in Zukunft weiter fortzusetzen. ${ }^{8}$ Dies zeigt sich deutlich bei einer Betrachtung des Investitionsprogrammes des Bundes ${ }^{9}$ für die Jahre 1999 bis 2002, das die Planungen des noch geltenden Bundesverkehrswegeplans auf Grund der außerordentlich ungünstigen Finanzsituation korrigiert. Von den in diesem Zeitraum $\mathrm{zu}$ realisierenden hochprioritären Investitionsmaßnahmen in Höhe von 64,5 Mrd. DM fließen genau die Hälfte, nämlich 32,1 Mrd. DM, in die neuen Bundesländer. ${ }^{10}$

\section{Bewertung der regionalen Verkehrsanbindungen in den alten und den neuen Bundesländern}

\subsection{Die Situation im Schienenverkehr}

\section{Die Güte der Verkehrsinfrastruktur Schiene}

Die Güte der Verkehrsinfrastruktur Schiene auf der Ebene von Stadt- und Landkreisen

Berechnet man die Güte der Verkehrsinfrastruktur Schiene in den Stadt- und Landkreisen in Deutschland in den Jahren 1990 und 1999, so lässt sich feststellen, dass die Masse der besonders schlechten regionalen Untersuchungseinheiten 1990 in den neuen Bundesländern lag. Mit Ausnahme der Kreise in Mecklenburg-Vorpommern gehörte praktisch der gesamte Rest der ehemaligen DDR zu jener Klasse, die eine besonders schlechte Verkehrsinfrastruktur auf der Schiene aufwies. 1990 zählten zur besten Kategorie nur acht regionale Untersuchungseinheiten; von ihnen aus war es möglich, mit mehr als $60 \mathrm{~km} / \mathrm{h}$ Luftliniengeschwindigkeit zu den übrigen Stadt- und Landkreisen in Deutschland zu fahren. Spitzenreiter war dabei Flensburg mit $64,1 \mathrm{~km} / \mathrm{h}$, gefolgt von Bremen mit 63,8 und Lörrach mit $63,5 \mathrm{~km} / \mathrm{h}$. Diese Werte liegen mehr als doppelt so hoch wie bei den schlechtesten drei Stadt- und Landkreisen, die durch Hildburghausen $(31,9 \mathrm{~km} \mathrm{~h})$, den Oberbergischen Kreis $(31,3 \mathrm{~km} / \mathrm{h})$ und den Kreis Sonneberg $(28,3 \mathrm{~km} / \mathrm{h})$ gebildet wurden.

Diese Situation hat sich in der Zwischenzeit deutlich geändert. Die regionalen Untersuchungseinheiten, die besonders schlecht erschlossen sind, zeigen jetzt kein West-Ost-Gefälle mehr, sondern lassen sich vor allem über topographische Gegebenheiten erklären; sie liegen vor allem im Sauerland, im Thüringer Wald und im Erzgebirge. Die besten regionalen Untersuchungseinheiten verteilen sich gleichmäßig zwischen West und Ost und liegen vor allem in der norddeutschen Tiefebene, aber auch am Oberrhein und im südlichen Bayern. Spitzenreiter ist erneut Flensburg mit $74,7 \mathrm{~km} / \mathrm{h}$, jetzt aber 'schon gefolgt von Berlin mit $74,1 \mathrm{~km} / \mathrm{h}$. Von den drei schlechtesten Kreisen liegen mit Westerwald $(37,1 \mathrm{~km} / \mathrm{h})$ und Oberberg $(34,5 \mathrm{~km} / \mathrm{h})$ zwei in Westdeutschland und nur einer, nämlich Annaberg mit $36,3 \mathrm{~km} / \mathrm{h}$, in Ostdeutschland.

Um die zwischen 1990 und 1999 stattgefundene Entwicklung noch deutlicher zu machen, ist in Abbildung 1 die Veränderung der Luftliniengeschwindigkeit in den Stadt- und Landkreisen Deutschlands dargestellt worden. Die regionalen Untersuchungseinheiten, deren Luftliniengeschwindigkeit besonders stark angestiegen ist, konzentrieren sich in Ostdeutschland und hier vor allem in einem breiteren Umfeld um die Schnellbahnstrecke Hannover-Berlin. Deutlich wird auch der Effekt der Neubaustrecke Hannover-Kassel.

Es bleibt festzuhalten: Im Jahr 1990 bestand ein deutliches Gefälle zwischen den Kreisen in West- und Ostdeutschland, das bis 1999 vollkommen verschwunden ist. Feststellbar ist nur noch ein Unterschied, der topographisch begründbar ist (Flachland versus Mittelgebirge) und der West- und Ostdeutschland in gleicher Weise trifft.

Die Güte der Verkehrsinfrastruktur Schiene auf der Ebene der Bundesländer

Die bisher auf der Ebene von Stadt- und Landkreisen durchgeführte Analyse soll jetzt in der Ebene der Bundesländer verdichtet werden, um die Ergebnisse überschaubarer, leichter interpretierbar und politisch relevanter zu machen. Die Landesergebnisse ergeben sich 


\section{Abbildung 1}

Veränderung der Güte der Verkehrsinfrastruktur Schiene in den Stadt- und Landkreisen Deutschlands zwischen 1990 und 1999 (Veränderung der Luftliniengeschwindigkeit in $\mathrm{km} / \mathrm{h}$ zu allen übrigen Kreisen in Deutschland; gravitationstheoretischer Ansatz)

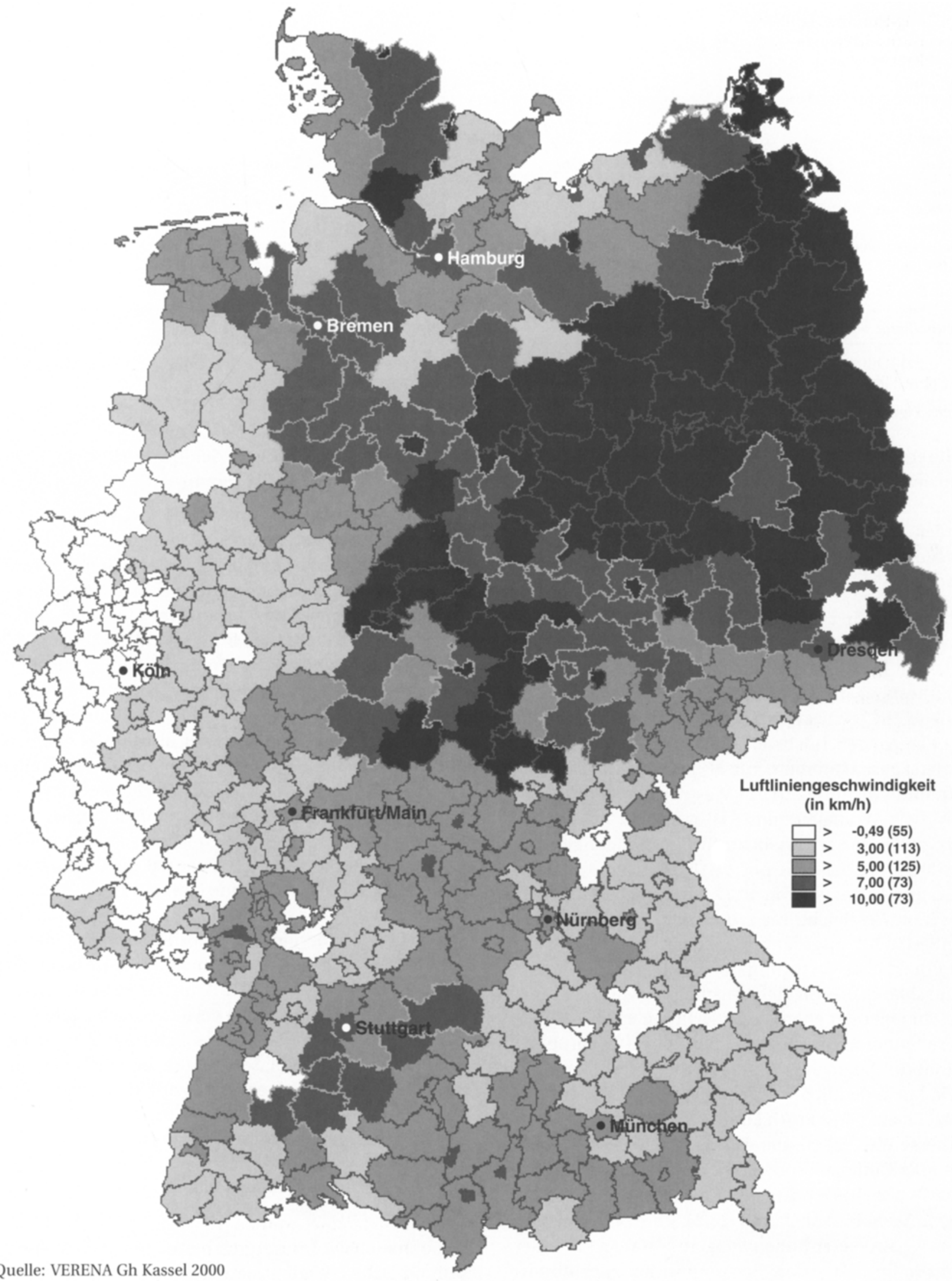


Tabelle 2

Güte der Verkehrsinfrastruktur Schiene auf der Ebene von Bundesländern

\begin{tabular}{|l|c|c|c|c|c|c|}
\hline & vl 90 & vl 99 & Veränderung & Varianz 90 & Varianz 99 & Veränderung \\
\hline Schleswig-Holstein/Hamburg & 56,19 & 64,29 & 8,10 & 24,34 & 32,53 & 8,19 \\
Niedersachsen/Bremen & 53,31 & 61,45 & 8,14 & 21,32 & 30,27 & 8,95 \\
Nordrhein-Westfalen & 45,64 & 48,84 & 3,20 & 26,93 & 33,44 & 6,51 \\
Hessen & 44,85 & 51,08 & 6,23 & $\mathbf{1 2 , 8 6}$ & 21,02 & 8,16 \\
Rheinland-Pfalz/Saarland & 46,49 & 50,14 & 3,65 & 15,57 & 20,95 & 5,38 \\
Baden-Württemberg & 46,67 & 52,47 & 5,80 & 32,74 & 29,88 & $-2,86$ \\
Bayern & 50,60 & 55,59 & 4,99 & 18,70 & 21,74 & 3,04 \\
alte Bundesländer (ohne West-Berlin) & 48,46 & 53,72 & 5,26 & 35,68 & 52,97 & 17,29 \\
Brandenburg/Berlin & 46,44 & 67,38 & 20,94 & 5,95 & 69,98 & 64,03 \\
Mecklenburg-Vorpommern & 52,01 & 61,51 & 9,50 & 14,57 & 17,96 & 3,39 \\
Sachsen & 41,18 & 48,99 & 7,81 & 12,80 & 31,68 & 18,88 \\
Sachsen-Anhalt & 42,75 & 54,82 & 12,07 & 12,29 & 25,64 & 13,35 \\
Thüringen & 40,17 & 49,58 & 9,41 & 15,04 & 18,77 & 3,73 \\
neue Bundesländer (inkl. West-Berlin) & 44,20 & 57,55 & 13,35 & 23,97 & 103,98 & 80,01 \\
\hline Deutschland insgesamt & 47,54 & 54,55 & 7,01 & 36,22 & 66,57 & 30,35 \\
\hline
\end{tabular}

$\mathrm{vl}=$ durchschnittliche Luftliniengeschwindigkeit der Kreise des jeweiligen Bundeslandes zu allen übrigen Kreisen in Deutschland (gravitationstheoretischer Ansatz)

Quelle: Eigene Berechnungen

dadurch, dass man das gewichtete arithmetische Mittel über jene Stadt- und Landkreise errechnet, die dem entsprechenden Bundesland angehören; die Gewichte werden dabei durch die Einwohnerzahlen gebildet. Tabelle 2 bringt die entsprechenden Ergebnisse zum Ausdruck.

Betrachten wir zunächst die Güte der Verkehrsinfrastruktur, die erneut durch die Luftliniengeschwindigkeit in $\mathrm{km} / \mathrm{h}$ zum Ausdruck gebracht wird. Im Jahr 1990 bestand die beste Verkehrsinfrastruktur auf der Schiene in Schleswig-Holstein/Hamburg, das mit $56,19 \mathrm{~km} / \mathrm{h}$ den höchsten Wert aufwies, gefolgt von Niedersachsen/Bremen mit 53,31 und MecklenburgVorpommern mit 52,01. In Westdeutschland bildeten Nordrhein-Westfalen und Hessen mit 45,60 und $44,85 \mathrm{~km} / \mathrm{h}$ das Schlusslicht; ihre Ausprägungen wurden aber von Sachsen mit 41,18, Sachsen-Anhalt mit 42,75 und Thüringen mit $40,17 \mathrm{~km} / \mathrm{h}$ noch deutlich unterboten. Vergleicht man die alten und die neuen Bundesländer insgesamt miteinander, so ergibt sich ein erheblicher Vorsprung für die alte Bundesrepublik Deutschland, die mit einer Ausprägung von $48,46 \mathrm{~km} / \mathrm{h}$ klar vor jener der ehemaligen DDR mit $44,20 \mathrm{~km} / \mathrm{h}$ lag. Diese Situation hat sich bis zum Jahre 1999 gründlich geändert. Die neuen Bundesländer führen nun mit $57,55 \mathrm{~km} / \mathrm{h}$ deutlich vor den alten Bundesländern, die lediglich auf $53,72 \mathrm{~km} / \mathrm{h}$ kommen. Profitiert von dieser Verbesserung haben alle ostdeutschen Länder, vor allem aber Brandenburg/Berlin, dessen Ausprägung um $45,08 \%$ angestiegen ist; auf den nächsten Rangplätzen folgen Sachsen-Anhalt mit 28,24 und Thüringen mit $23,40 \%$. Mit dieser hohen Steigerungsrate ist Brandenburg/Berlin nun an die Spitzenposition aller Bundes- länder gerückt; ihm folgen Schleswig-Holstein/Hamburg und Mecklenburg-Vorpommern.

Berechnet man die Varianz der Güte der Verkehrsinfrastruktur, so lässt sich erkennen, ob die Erschließung in der Fläche relativ gleichmäßig ist oder sich nur auf wenige ausgewählte Raumpunkte, i.d.R. besonders bedeutsame Zentren, konzentriert. Im Jahr 1990 zeigen sich in den westdeutschen Bundesländern höhere Varianzen als in den ostdeutschen. So ist Baden-Württemberg mit 32,74 Spitzenreiter, während Brandenburg/ Berlin mit 5,95 auf der letzten Rangposition liegt. Die flächenmäßige Erschließung war also in der ehemaligen DDR 1990 signifikant gleichmäßiger als in der alten Bundesrepublik; dort war die Verkehrsinfrastruktur Schiene zwar im Durchschnitt deutlich besser, konzentrierte sich aber wesentlich stärker auf die großen Zentren. Diese Situation hat sich - wie die Güte der Verkehrsinfrastruktur - bis zum Jahr 1999 ins Gegenteil verkehrt. Besonders hohe Varianzen zeigen sich nun in den neuen Bundesländern, speziell in Brandenburg/ Berlin, aber auch in Sachsen und Sachsen-Anhalt. Der Ausbau der Verkehrsinfrastruktur hat zwar die neuen Bundesländer deutlich nach vorne gebracht, aber auch die Unterschiede im Raum erheblich vergrößert. Insbesondere die Verbindungsqualitäten zwischen den groBen Zentren sind aufgewertet worden, während dies für die Erschließung der Zentren gegenüber dem Umland nicht in gleicher Weise der Fall ist.

\section{Die Güte der oberzentralen Verbindungen im Schienenverkehr}

Bisher haben wir Indikatoren betrachtet, die auf einem summarischen Wert basieren; es wurden von jedem 
Kreis aus die Luftliniengeschwindigkeiten zu allen übrigen Stadt- und Landkreisen in Deutschland berechnet und aus diesen 439 Ausprägungen ${ }^{11}$ ein gewichtetes arithmetisches Mittel gebildet. Nun sollen einzelne Verbindungen zwischen ausgewählten regionalen Untersuchungseinheiten betrachtet werden; da es wenig sinnvoll erscheint, Verbindungen zwischen peripheren ländlichen Regionen mit jenen zwischen bedeutenden Zentren zu vergleichen, beschränkt sich diese Betrachtung auf Interrelationen zwischen Oberzentren. Besonders gute Verbindungen fanden sich 1990 fast ausschließlich in Westdeutschland, so vor allem zwischen dem Ruhrgebiet auf der einen und den Zentren der norddeutschen Tiefebene auf der anderen Seite sowie in der Oberrheinischen Tiefebene. Spitzenreiter war die Verbindung Fulda-Würzburg mit einer Luftliniengeschwindigkeit von $130,5 \mathrm{~km} / \mathrm{h}$, gefolgt von Bielefeld-Dortmund mit 127,1 und Osnabrück-Bremen mit $121,2 \mathrm{~km} / \mathrm{h}$. Die schlechten Ausprägungen basieren zum einen auf der Notwendigkeit, wegen topographischer Situationen große Umwege fahren zu müssen; dies gilt etwa für die Verbindung von Bremerhaven nach Wilhelmshaven, die wegen der sie trennenden Wesermündung über Bremen miteinander

\section{Abbildung 2a)}

Oberzentrale Verbindungen 1999 mit mehr als $100 \mathrm{~km} / \mathrm{h}$ Luftliniengeschwindigkeit auf der Schiene

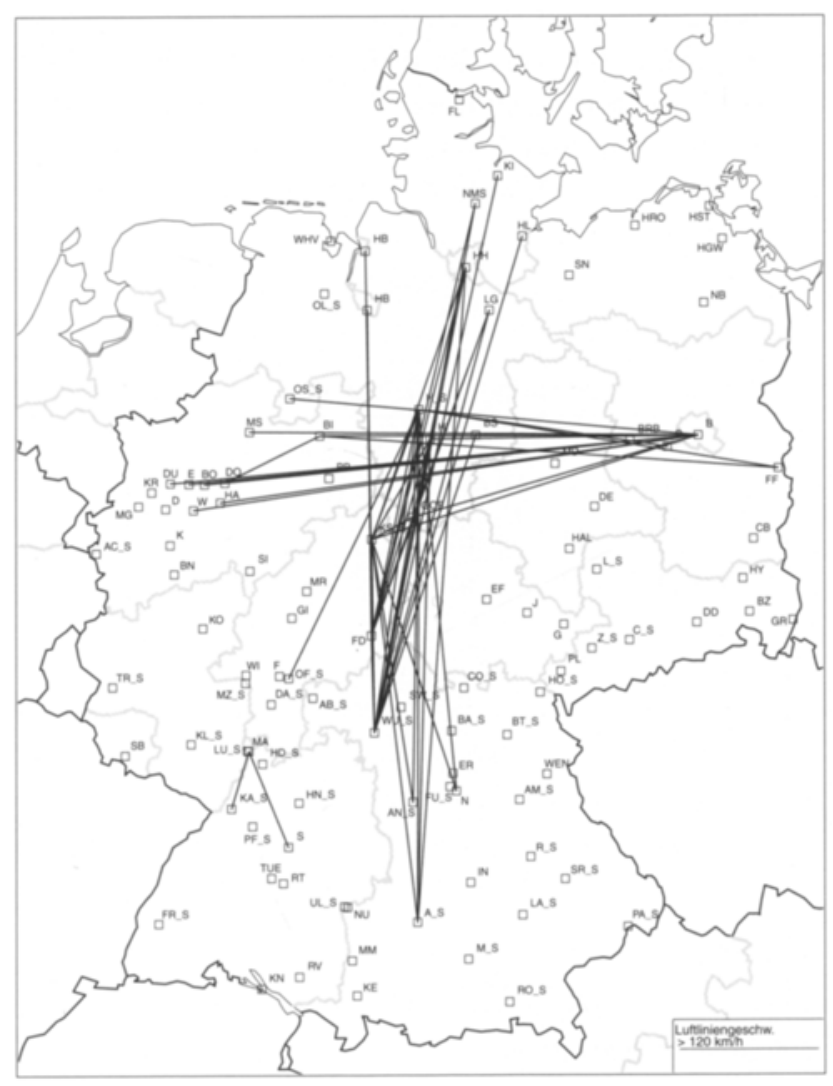

Quelle: VERENA Gh Kassel 2000 verbunden sind, und für Beziehungen zwischen Oberzentren Sachsens und Bayerns, bei denen es wenig sinnvoll ist, das wenig leistungsfähige tschechische Eisenbahnnetz zu nutzen. Zum anderen zeichnet für die schlechte Verbindungsqualität aber auch eine unzureichende Verkehrsinfrastruktur verantwortlich. So konnte 1990 auf den Verbindungen Hof-Jena, Hof-Erfurt und Plauen-Jena nur mit einer Luftliniengeschwindigkeit von wenig mehr als $20 \mathrm{~km} / \mathrm{h}$ gefahren werden. Diese Geschwindigkeit lag damit nur bei einem Sechstel der besten miteinander verbundenen Oberzentren in Westdeutschland.

Abbildung 2a) und Abbildung 2b) bringen denselben Tatbestand für das Jahr 1999 zum Ausdruck. Als besonders gut werden dabei jene oberzentralen Verbindungen dargestellt, bei denen die Luftliniengeschwindigkeit mehr als $120 \mathrm{~km} / \mathrm{h}$ beträgt. Abbildung 2a) zeigt zum einen den großen Erschließungseffekt der Neubaustrecken Hannover-Kassel-Würzburg und StuttgartMannheim, zum anderen aber auch, dass mittlerweile eine sehr leistungsfähige Magistrale vom Ruhrgebiet über Hannover nach Berlin existiert. Die Menge der schlechten Verbindungen (Luftliniengeschwindigkeit

Abbildung 2b)

Oberzentrale Verbindungen 1999 mit weniger als $40 \mathrm{~km} / \mathrm{h}$ Luftliniengeschwindigkeit auf der Schiene

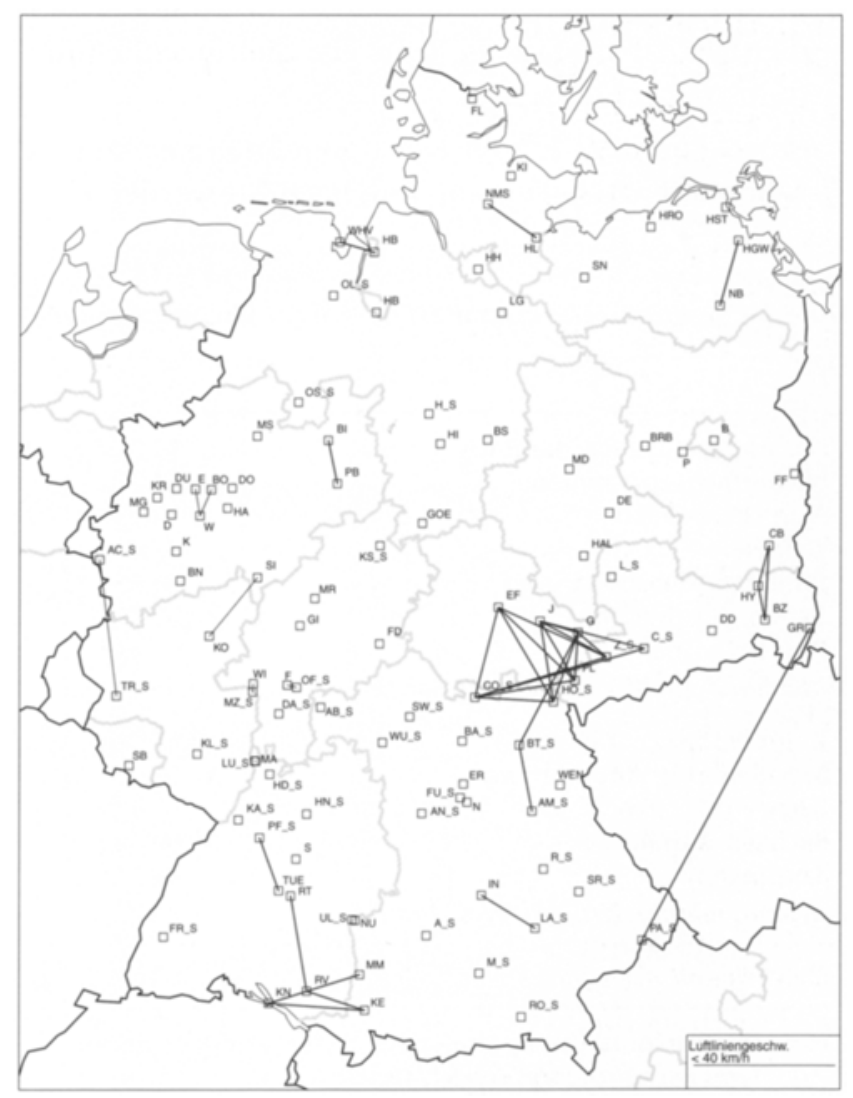

Quelle: VERENA Gh Kassel 2000 
weniger als $40 \mathrm{~km} / \mathrm{h}$ ) ist zwischen 1990 und 1999 von 115 auf 40 zurückgegangen. Sie konzentrieren sich auf das Dreiländereck Bayern, Sachsen und Thüringen.

\subsection{Die Situation im Straßenverkehr}

Im Hinblick auf den Straßenverkehr sind Aussagen zu treffen, die sich deutlich von jenen zum Schienenverkehr unterscheiden.

- Zwar war 1990 auch im Straßenverkehr ein OstWest-Gefälle feststellbar, das jedoch merklich geringer war als im Schienenverkehr. Im Westen bestand zwar ein deutlich leistungsfähigeres Autobahnnetz, das jedoch häufig überlastet war (und ist). Im Osten war das stark überalterte Straßennetz weniger belastet.

- Zwischen 1990 und 1999 sind nur geringfügige Verbesserungen der Luftliniengeschwindigkeit feststellbar. Im Vergleich zum Ausbau des Schienenhinkt der Ausbau des Straßennetzes deutlich nach. Die qualitativen Defizite im Osten sowie die Überlastung im Westen halten an. Die Differenz zwischen Ost und West ist praktisch gleich geblieben.

- Waren selbst auf der Ebene von Bundesländern beim Schienenverkehr erhebliche interregionale Erschließungsunterschiede feststellbar, so sind die errechneten Varianzen beim Straßenverkehr erheblich niedriger. Die Erschließung durch die Straße ist ein deutlich ubiquitäreres Gut als die Erschließung durch die Schiene.

- Da in den 90er Jahren Neubaumaßnahmen nur in sehr begrenztem Umfang durchgeführt worden sind und sich die Verbesserungen weitgehend auf eine Wiederherstellung der durch den Zweiten Weltkrieg unterbrochenen Verbindungen zwischen Ost und West sowie den Ausbau von Straßen konzentrierten, hat sich die Varianz zwischen den Stadt- und Landkreisen in Deutschland kaum geändert; diese Aussage gilt sowohl für den Osten als auch für den Westen. Einzelheiten können der Tabelle 3 entnommen werden.

Abbildung 3 lässt erkennen, welche Verbindungen zwischen Oberzentren im Augenblick unzureichend sind; zwischen den entsprechenden Quell- und Zielorten kann nur mit einer Luftliniengeschwindigkeit von weniger als $50 \mathrm{~km} / \mathrm{h}$ gefahren werden. Besonders schlechte Verbindungen basieren darauf, dass

- sehr große Umwege gefahren werden müssen. Dies ist etwa in Küstenregionen der Fall.

- das bestehende Verkehrswegenetz überlastet ist. Eine solche Aussage gilt z.B. für das Rhein-Ruhrsowie das Rhein-Main-Gebiet.

- Oberzentren nur durch wenig leistungsfähige Straßen miteinander verbunden sind. Hier müssen Bundes- und Landstraßen genutzt oder über Autobahnen große Umwege gefahren werden. Beispiele für solche Verbindungen sind etwa Marburg-Paderborn und Kassel-Siegen. Die in Ostdeutschland erkennbaren Defizite werden weitestgehend abgebaut sein, wenn die Verkehrsprojekte Deutsche Einheit Realität geworden; sind. Eine besondere Rolle hierbei spielen die beabsichtigten Autobahnen Halle-Magdeburg, Lübeck-Stettin und Erfurt-Coburg.

Tabelle 3

Güte der Verkehrsinfrastruktur Straße auf der Ebene von Bundesländern

\begin{tabular}{|l|c|c|c|r|r|r|}
\hline & vl 90 & vl 99 & Veränderung & Varianz 90 & Varianz 99 & Veränderung \\
\hline Schleswig-Holstein/Hamburg & 66,36 & 66,54 & 0,18 & 8,85 & 8,88 & 0,03 \\
Niedersachsen/Bremen & 64,41 & 64,80 & 0,39 & 10,40 & 10,54 & 0,14 \\
Nordrhein-Westfalen & 64,72 & 64,97 & 0,25 & 12,12 & 12,83 & 0,71 \\
Hessen & 63,64 & 64,33 & 0,69 & 8,23 & 7,58 & $-0,65$ \\
Rheinland-Pfalz/Saarland & 63,72 & 64,14 & 0,42 & 8,48 & 8,65 & 0,17 \\
Baden-Württemberg & 63,97 & 64,15 & 0,18 & 11,66 & 11,77 & 0,11 \\
Bayern & 64,44 & 65,07 & 0,63 & 8,80 & 8,50 & $-0,3$ \\
alte Bundesländer (ohne West-Berlin) & 64,44 & 64,82 & 0,38 & 10,76 & 10,82 & 0,06 \\
Brandenburg/Berlin & 67,42 & 67,69 & 0,27 & 7,42 & 7,27 & $-0,15$ \\
Mecklenburg-Vorpommern & 61,95 & 62,34 & 0,39 & 6,21 & 6,31 & 0,1 \\
Sachsen & 61,47 & 62,42 & 0,95 & 9,44 & 10,64 & 1,2 \\
Sachsen-Anhalt & 60,49 & 61,42 & 0,93 & 7,13 & 8,56 & 1,43 \\
Thüringen & 60,15 & 60,57 & 0,42 & 7,26 & 7,74 & 0,48 \\
neue Bundesländer (inkl. West-Berlin) & 63,20 & 63,78 & 0,58 & 17,22 & 16,53 & $-0,69$ \\
\hline Deutschland insgesamt & 64,17 & 64,59 & 0,42 & 12,42 & 12,25 & $-0,17$ \\
\hline vl durchschnittliche Luftliniengeschwindigkeit der Kreise des jeweiligen Bundeslandes zu allen übrigen Kreisen in Deutschland \\
(gravitationstheoretischer Ansatz)
\end{tabular}

Quelle: Eigene Berechnungen 
Abbildung 3

Oberzentrale Verbindungen 1999 mit weniger als $50 \mathrm{~km} / \mathrm{h}$ Luftliniengeschwindigkeit auf der Straße

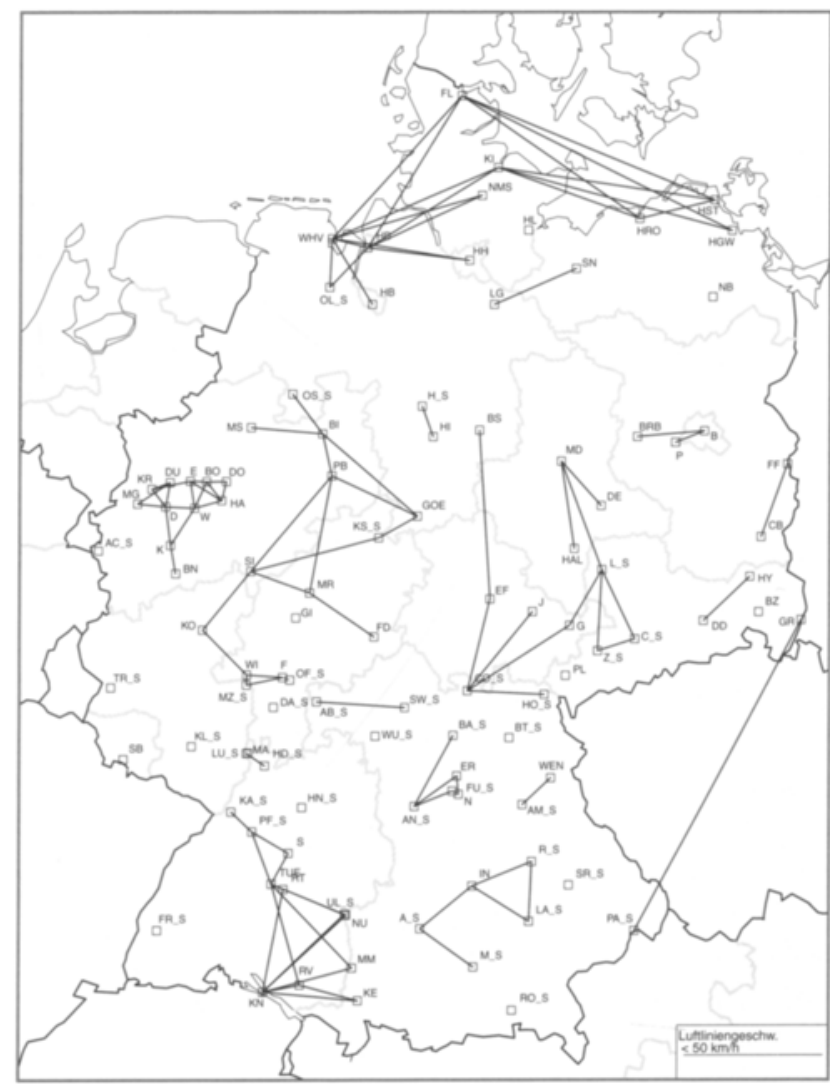

\section{Zusammenfassung der Ergebnisse}

Neben anderen Faktoren haben die politischen Veränderungen in den Ost-West-Beziehungen seit Ende 1989 und die Wiedervereinigung Deutschlands zu neuen Anforderungen an die Verkehrspolitik geführt. So war und ist es politisches Ziel, die Verkehrsinfrastruktur in den neuen Bundesländern erheblich zu verbessern, um sie nicht zu einem Engpass für die wirtschaftliche Entwicklung werden zu lassen.

Die durchgeführten empirischen Arbeiten beschäftigen sich mit der Frage, inwieweit es in den zehn Jahren seit Grenzöffnung gelungen ist, die Unterschiede in der Verkehrsinfrastruktur zwischen Ost und West zu verringern oder gar zu beseitigen. Dabei zeigt sich, dass eine erhebliche Angleichung im Schienenverkehr stattgefunden hat; die Stadt- und Landkreise in Ostdeutschland sind heute bereits im Durchschnitt besser an das Schienennetz angeschlossen als die entsprechenden regionalen Untersuchungseinheiten im Westen. Dagegen sind die eingetretenen Veränderungen im Straßenverkehr minimal; die Unterschiede zwischen Ost und West bestehen fast unverändert fort.

\section{Anmerkungen}

(1)

Unter Verantwortung und Leitung des Mitverfassers, Herrn Dr. Klaus Horn

(2)

Nach Auffassung der Verfasser ist dieser Indikator gängigen Kennziffern wie Länge der Eisenbahnstrecken, Fläche, Vorhandensein eines IC-Bahnhofs, Anbindung an das Hochgeschwindigkeitsnetz usw. überlegen, da es zum einen nicht auf die physische Präsenz von Infrastruktur, sondern der Erschließungsqualität ankommt und zum anderen von den in einer Region vorhandenen Eisenbahnstrecken auch die Einwohner anderer Wirtschaftsräume profitieren, indem sie sie etwa bei Durchfahrten nutzen.

(3)

Ein hervorragender Überblick zu den vorliegenden ökonometrischen Untersuchungen findet sich bei Hofmann, Ulrich: Produktivitätseffekte der öffentlichen Infrastruktur. - Frankfurt a.M. 1996, insbes. S. $15 \mathrm{ff}$.

(4)

Deutsches Institut für Wirtschaftsforschung (DIW) (Hrsg.): Immer noch Nachholbedarf bei der Verkehrsinfrastruktur in Ostdeutschland. In: Wochenbericht, 63. Jg. (1996) 50 v. 12.12.1996, S. $805-815$

(5)

ifo-Institut (Hrsg.): Investitionsaktivitäten westdeutscher Unternehmen in der ehemaligen DDR. In: ifo-Schnelldienst (1991) 12, S. $6 \mathrm{ff}$.

(6)

dass. (Hrsg.): Infrastrukturengpässe in den neuen Bundesländern. In: ifo-Schnelldienst (1991) 6, S. 3 ff.

(7)

Deutsches Institut für Wirtschaftsforschung (DIW) (Hrsg.): Immer noch Nachholbedarf . . , a.a.O. [siehe Anm. (4)], S. 805-815

(8)

Auf die Intentionen des Bundesverkehrswegeplans wurde bereits im Kapitel „Aufgabenstellung der Untersuchung“ hingewiesen.

(9)

Vgl. Bundesministerium für Verkehr, Bau- und Wohnungswesen (Hrsg.): Investitionsprogramm für den Ausbau der Bundesschienenwege, Bundesfernstraßen und Bundeswasserstraßen in den Jahren 1999 bis 2002. - Berlin 1999

(10)

o.V.: Investitionsprogramm Bundesverkehrswege 1999-2002. In: Internationales Verkehrswesen, 52. Jg. (2000) Jan.: Febr., S. 7

(11)

Die Menge der Stadt- und Landkreise in Deutschland beträgt 440.

Prof.Dr. Hans-Friedrich Eckey

Dr. Klaus Horn

Universität Gh Kassel

Fachbereich 07: Wirtschaftswissenschaften

Postfach 101380

34109 Kassel

E-Mail: eckey@wirtschaft.uni-kassel.de 